\title{
Existence of torsional surface waves in an earth's crustal layer lying over a sandy mantle
}

\author{
Sumit Kumar Vishwakarma ${ }^{1,2, *}$ and Shishir Gupta ${ }^{1}$ \\ ${ }^{1}$ Department of Applied Mathematics, Indian School of Mines, Dhanbad 826 004, India. \\ ${ }^{2}$ Department of Mathematics, Birla Institute of Technology and Science-Pilani, \\ Hyderabad 500 078, India. \\ ${ }^{*}$ Corresponding author.e-mail: sumitkumar@hyderabad.bits-pilani.ac.inｓumo.ism@ismu.ac.in
}

This paper aims to study the dispersion of torsional surface waves in a crustal layer being sandwiched between a rigid boundary plane and a sandy mantle. In the mantle, rigidity and initial stress vary linearly while density remains constant. Dispersion relation has been deduced in a closed form by means of variable separable method in the form of Whittaker function. The velocity equation for isotropic layer over a homogeneous half-space has been obtained which coincides with the standard result of Love wave under the effect of rigid boundary.

\section{Introduction}

The earth is layered solid under high initial stresses. Due to atmosphere, variation of temperature, gravitating pull, slow process of creep and pressure due to overburdened layer, a large quantity of stresses (may be critical initial stresses) are stored in the layer of the earth. Artificially generated seismic waves provide information about the configuration of the rock layer for oil exploration, ground water prospecting and on smaller scale information as to the rigidity of shallow layer for engineering purposes. A straight mathematical attack may produce explicit or numerical solutions even for rather complex solid media. The mathematical expression provides the bridge between modelling results and field application. Any disturbance in earth's interior may serve as the basic reason of seismic wave propagation. The theoretical study of wave propagation consists of finding the solution of a partial differential equation or a system of partial differential modelling under initial and boundary conditions. The propagation of seismic waves through the earth's interior is governed exactly by mathematical laws similar to the laws of light waves in optics. If the propagation velocities and other elastic properties were uniform throughout the earth, seismic waves would radiate from the focus of the earthquake in all the directions through the earth along rectilinear path or rays. In general, however, the wave velocity increases with depth and consequently, seismic rays are not straight lines but lines curved with the concave side upward providing the shortest time past through the earth.

Earth is a gravitating initially stressed medium due to the presence of overburdened layers. The presence of gravity field and internal friction of dry sandy material will have effect on the propagation of the waves. The acceleration due to gravity $g$ has a greater role to play in studying the dynamic and static problems of the earth. Kepceler (2010) has studied torsional wave dispersion relations in a pre-stressed bi-material compounded cylinder with an imperfect interface. Propagation of torsional surface waves in a homogeneous layer of finite

Keywords. Torsional wave; mantle; Biot's gravity parameter; graphical user interface. 
thickness over an initially stressed heterogeneous half-space have been recently studied by Gupta et al. (2012a) whereas propagation of torsional surface waves in dry sandy medium under gravity has been studied by Dey et al. (1998). References can be made to Dey and Sarkar (2002); Selim (2007); Ozturk and Akbbarov (2009); Akbarov et al. (2011) and Gupta et al. (2012b, c) for their excellent contribution in investigating torsional surface wave in various mediums under various circumstances. Behaviour of torsional surface wave in an elastic layer with void pores has been studied by Dey et al. (2003) whereas torsional wave propagation in a thin rectangular domain using asymptotic approach has been handled by Davini et al. (2008). Golamhossen (2000) pointed out the propagation of waves in an elastic cylinder with voids while a detailed study has been made in axially symmetric problems for a porous elastic solid by Lesan and Nappa (2003). Some authors investigated the effect of nonlinear variations in the elastic moduli, density and initial stress with various geo-media. Dey et al. (1996) discussed the possibility of propagation of torsional surface waves in a nonhomogeneous elastic medium with polynomial variation of rigidity and density, i.e., $\mu=\mu_{0}(1+a z)^{2 n}$ and $\rho=\rho_{0}(1+a z)^{m}$. They found that inhomogeneity parameter being non-linearly associated with rigidity and density has a prominent effect on torsional surface wave. Very recently, Chattaraj et al. (2011) investigated the propagation of torsional surface wave in anisotropic poroelastic medium under initial stress, where he has taken quadratic variation in the directional rigidities, density and initial stress, i.e., $N=N_{0}(1+\alpha z)^{2}, L=L_{0}(1+\alpha z)^{2}$, $\rho=\rho_{0}(1+\alpha z)^{2}, P=P_{0}(1+\alpha z)^{2}$. They found that the effect of quadratic variation is more pronouncing at higher frequency. Although the nature and characteristic of the curves for nonlinear variations are similar to that being plotted for linear variation in the present problem; on the basis of the studies being made by the authors in past, it can be concluded that the effect of quadratic variation on the phase velocity of torsional surface wave may come out to be similar to that of the linear one, and has no special contribution or emphasize over the torsional surface wave. The reason for this may lie to the fact that the basic characteristic of surface wave is that it decreases as depth increases. Therefore $z^{2}$ and higher power of $z$ have been neglected and only linear variation has been preferred in the present problem. The works done by Georgiadis et al. (2000); Quintanilla (2001); Midya (2004); Arora and Tomar (2007) and Gupta et al. (2013) cannot be overlooked as their contributions are commendable towards seismic wave propagation.
So far, it has been found that the effect of gravity on the propagation of torsional surface wave in an inhomogeneous anisotropic layer has remained unattempted. Therefore, in the present paper, an attempt has been made to study the propagation of torsional surface waves in an anisotropic inhomogeneous layer over gravitating dry sandy mantle. Torsional surface wave is a wave with amplitude decaying exponentially with distance from the free surface. Inside the earth, a very hard layer (also known as 'rigid') is present. Since the composition of the earth is heterogeneous including a very hard layer, the inhomogeneous medium and the rigid interface play significant roles in the propagation of the seismic waves. A dry sandy mantle may be defined as a half-space consists of sandy particles retaining no moistures or water vapours, the characteristic of which has been defined by $\eta$ known as sandy parameter.

For the present study, the heterogeneity taken is caused by variation in directional rigidities and density in the layer whereas heterogeneity taken in the mantle is caused by variation in rigidity and initial stress. The crust region of our planet is composed of various heterogeneous layers with different geological parameters. As pointed out by Bullen (1940), the density inside the earth varies at different rates with different layers within the earth. He approximated density law inside the earth as a quadratic polynomial in depth parameter for 413$984 \mathrm{~km}$ depth. For depth from $984 \mathrm{~km}$ to the central core, Bullen approximated the density as a linear function of depth parameter. Sari and Salk (2002) took the variation in the density of sediments with depth as a hyperbolic function. Likewise, different authors have taken different forms of variation, like harmonic, linear, quadratic, exponential, etc., for simulating the variation in density and other geological parameters inside the earth. Therefore, in the present paper, variations taken in the layer are $N=N_{0} e^{z / \alpha}, L=L_{0} e^{z / \alpha}, \rho=$ $\rho_{0} e^{z / \alpha}$, whereas variation taken in the mantle are $\mu=\mu_{1}(1+a z), \quad P=P_{1}(1+b z), \quad \rho=\rho_{1}$, where $\alpha$ is a constant having dimension equal to length and $a, b$ are constants having dimension equal to inverse of length. ' $z$ ' is a vertical coordinate axis, positive downward having origin at the interface (as shown in figure 1). It may also be called as depth coordinate. In the half-space, rigidity varies linearly whereas density remains constant throughout; this is called Gibson half-space (Vardoulakis 1984). Keeping this in view, an attempt has been made to find out whether such an inhomogeneous layer can allow torsional surface wave to propagate through it when lower Gibson half-space (mantle) is dry and sandy under the effect of gravitation. It is observed that the phase velocity of torsional surface wave increases 


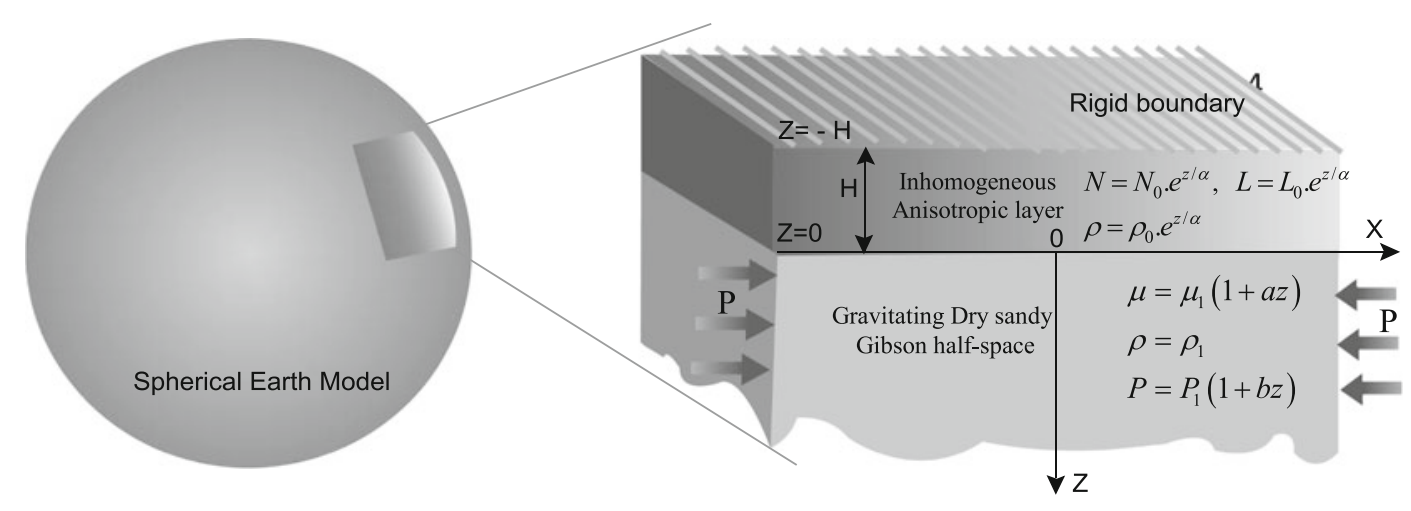

Figure 1. Three-dimensional geometry of the problem.

with the increase of compressive initial stress while decreases as the tensile initial stress decreases. It has also been found that the phase velocity increases as the inhomogeneity parameter associated with rigidity and the initial stress increases while decreases as the inhomogeneity parameter associated with directional rigidity and density of the layer increases. It is observed that the presence of gravity field always allow the torsional surface wave to propagate. It has also been concluded that the torsional surface wave propagates more smoothly in the layer when the lower half-space is elastic in comparison to dry sandy half-space. Further, anisotropy has also much effect in enhancing the velocity of torsional surface wave.

The present study of the dispersion of torsional waves in an anisotropic inhomogeneous crustal layer over a sandy mantle can be used to refine our knowledge of crustal and sub-crustal region near the earth surface. This study may also be useful for the study of seismic waves generated by artificial explosions. Effect of torsional waves cannot be ignored in assessment of damage caused by earthquake waves. Hence, the present study with the assumed variation in rigidity, density and stress may be useful in predicting nature of torsional surface wave in inhomogeneous geo-media.

\section{Statement of the problem}

To study the torsional surface waves, a cylinder coordinate system is introduced with $z$-axis towards the interior of the gravitating dry sandy Gibson half-space. A Gibson half-space is one where rigidity varies linearly while density remains constant. The study has been made to get the dispersion equation of torsional surface wave in an inhomogeneous anisotropic layer of thickness $H$, over a sandy half-space under the effect of gravity, initial stress and rigid boundary as shown in figure 1 . The origin of the coordinate system is located at the surface of the half-space at the centre of a circular region.

\section{Solution}

\subsection{Solution of the inhomogeneous anisotropic layer}

If $r$ and $\theta$ be radial and circumferential coordinates respectively, and if the wave travels along the radial direction only, the equation of motion in the layer may be written as (Biot 1965):

$$
\frac{\partial \sigma_{r \theta}}{\partial r}+\frac{\partial \sigma_{z \theta}}{\partial z}+\frac{2}{r} \sigma_{r \theta}=\rho \frac{\partial^{2} v}{\partial t^{2}}
$$

where $v(r, z, t)$ is the displacement along $\theta$ direction and $\rho$ is the density.

For a non-homogeneous anisotropic elastic medium, the stresses are related to strain by

$$
\sigma_{r \theta}=2 N e_{r \theta}, \quad \sigma_{z \theta}=2 L e_{z \theta}
$$

where $e_{r \theta}=\frac{1}{2}\left(\frac{\partial v}{\partial r}-\frac{v}{r}\right), e_{z \theta}=\frac{1}{2} \frac{\partial v}{\partial z} . N$ and $L$ are directional rigidities of the medium along $r$ and $z$ directions, respectively.

Using the above relations, equation (1) takes the form

$$
N\left(\frac{\partial^{2} v}{\partial r^{2}}-\frac{v}{r^{2}}+\frac{1}{r} \frac{\partial v}{\partial z}\right)+\frac{\partial}{\partial z}\left(L \frac{\partial v}{\partial z}\right)=\rho \frac{\partial^{2} v}{\partial t^{2}} .
$$

The solution of equation (3) when wave propagate along radial direction with amplitude of displacement as a function of depth may be taken as:

$$
v=V(z) J_{1}(k r) e^{i \omega t},
$$

$\omega$ being circular frequency of the wave where $V(z)$ is the solution of

$$
\frac{d^{2} V(z)}{d z^{2}}+\frac{1}{L} \frac{d L}{d z} \frac{d V}{d z}-\frac{k^{2} N}{L}\left(1-\frac{c^{2} \rho}{N}\right)=0
$$


where $c=\omega / k$ is the velocity of the propagation of torsional surface wave and $J_{1}$ is the Bessel's function of first kind and of order one.

On substituting, $V=V_{1} / \sqrt{L}$ in equation (5), we get:

$$
\begin{gathered}
\frac{d^{2} V_{1}}{d z^{2}}-\frac{1}{2 L}\left\{\frac{d^{2} L}{d z^{2}}-\frac{1}{2 L}\left(\frac{d L}{d z}\right)^{2}\right\} V_{1} \\
=\frac{k^{2} N}{L}\left(1-\frac{c^{2} \rho}{N}\right) .
\end{gathered}
$$

Now, the variations in directional rigidities (elastic moduli) and density in the layer has been taken as:

$$
N=N_{0} e^{z / \alpha}, \quad L=L_{0} e^{z / \alpha}, \quad \rho=\rho_{0} e^{z / \alpha}
$$

Using equation (7), equation (6) becomes:

$$
\frac{d^{2} V_{1}}{d z^{2}}-m_{1}^{2} V_{1}=0
$$

where $m_{1}^{2}=k^{2}\left\{\frac{1}{4 \alpha^{2} k^{2}}+\frac{N_{0}}{L_{0}}\left(1-\frac{c^{2}}{c_{0}^{2}}\right)\right\}$ and $c_{0}=\sqrt{N_{0} / \rho_{0}}$ is the shear wave velocity in the layer.

The solution of equation (8) is given by

$$
V(z)=A_{1} e^{-m_{1} z}+A_{2} e^{m_{1} z}
$$

where $A_{1}$ and $A_{2}$ are arbitrary constants and hence the displacement in the upper inhomogeneous anisotropic layer is given by

$$
\begin{aligned}
v= & v_{0}(\text { say })=\left\{A_{1} e^{-\left(m_{1}+\frac{1}{2 \alpha}\right) z}+A_{2} e^{\left(m_{1}-\frac{1}{2 \alpha}\right) z}\right\} \\
& \times \frac{J_{1}(k r)}{\sqrt{L_{0}}} e^{i \omega t} .
\end{aligned}
$$

\subsection{Gravitating dry sandy mantle}

The dynamical equation of motion for the initially stressed dry sandy half-space under the effect of gravity as given by Biot (1965) may be written as:

$$
\begin{gathered}
\frac{\partial \sigma_{r \theta}}{\partial r}+\frac{\partial \sigma_{z \theta}}{\partial z}+\frac{2 \sigma_{r \theta}}{r}+\frac{\partial}{\partial z}\left\{(P-\rho g z) e_{z \theta}\right\} \\
-\rho g z \frac{\partial}{\partial r}\left\{\frac{1}{2}\left(\frac{\partial v}{\partial r}+\frac{v}{r}\right)\right\}=\rho \frac{\partial^{2} v}{\partial t^{2}}
\end{gathered}
$$

where $v(r, z, t)$ is the displacement along $\theta$ direction, $\rho$ is the density, $g$ is the acceleration due to gravity and $P$ is the compressive initial stress along $r$ direction. We also have $N=\eta \mu$, where $\eta$ is the sandy parameter and $\mu$ is the modulus of rigidity.

The inhomogeneity in the half-space is taken as:

$$
\mu=\mu_{1}(1+a z), \quad \rho=\rho_{1}, \quad P=P_{1}(1+b z) .
$$

Using relation $\sigma_{r \theta}=2 N e_{r \theta}$ and $\sigma_{z \theta}=2 N e_{z \theta}$ in equation (10), we get:

$$
\begin{aligned}
& \left\{\eta \mu_{1}(1+a z)-\frac{\rho g z}{2}\right\}\left\{\frac{\partial^{2} v}{\partial r^{2}}-\frac{v}{r^{2}}+\frac{1}{r} \frac{\partial v}{\partial r}\right\} \\
& +\left[\eta \mu_{1}(1+a z)+\frac{1}{2}\left\{P_{1}+\left(P_{1} b-g \rho\right) z\right\}\right] \frac{\partial^{2} v}{\partial z^{2}} \\
& +\left\{\eta \mu_{1} a+\frac{1}{2}\left(P_{1} b-\rho g\right)\right\} \frac{\partial v}{\partial z}=\rho \frac{\partial^{2} v}{\partial t^{2}}
\end{aligned}
$$

We assume the solution of equation (12) in the form of

$$
v=V(z) J_{1}(k r) e^{i \omega t}
$$

where $V(z)$ be solution of the equation given by

$$
\begin{aligned}
& \frac{d^{2} V}{d z^{2}}+\frac{\left\{\eta \mu_{1} a+\frac{1}{2}\left(P_{1} b-\rho g\right)\right\}}{\left[\eta \mu_{1}(1+a z)+\frac{1}{2}\left\{P_{1}+\left(P_{1} b-g \rho\right) z\right\}\right]} \\
& \frac{d V}{d z}-\frac{k^{2}\left\{\eta \mu_{1}(1+a z)-\frac{\rho g z}{2}\right\}}{\left[\eta \mu_{1}(1+a z)+\frac{1}{2}\left\{P_{1}+\left(P_{1} b-g \rho\right) z\right\}\right]} \\
& \times\left[1-\frac{\rho \omega^{2}}{k^{2}\left\{\eta \mu_{1}(1+a z)-\frac{\rho g z}{2}\right\}}\right] V=0 .
\end{aligned}
$$

Putting, $V(z)=\left(\varphi(z) /\left[\eta \mu_{1}(1+a z)+\frac{1}{2}\left\{P_{1}+\right.\right.\right.$ $\left.\left.\left.\left(P_{1} b-g \rho\right) z\right\}\right]^{1 / 2}\right)$ in equation (14), we get

$$
\begin{aligned}
& \frac{d^{2} \varphi}{d z^{2}}+\frac{1}{4} \frac{\left\{\eta \mu_{1} a+\frac{1}{2}\left(P_{1} b-g \rho\right)\right\}^{2} \varphi(z)}{\left[\eta \mu_{1}(1+a z)+\frac{1}{2}\left\{P_{1}+\left(P_{1} b-g \rho\right) z\right\}\right]^{2}} \\
& -\frac{k^{2}\left[\eta \mu_{1}(1+a z)-\frac{\rho g z}{2}\right]}{\left[\eta \mu_{1}(1+a z)+\frac{1}{2}\left\{P_{1}+\left(P_{1} b-g \rho\right) z\right\}\right]} \\
& \quad \times\left[1-\frac{\rho \omega^{2}}{k^{2}\left\{\eta \mu_{1}(1+a z)-\frac{\rho g z}{2}\right\}}\right] \varphi(z)=0
\end{aligned}
$$

Now, substituting $\varphi(z)=\varphi_{1}(\delta)$ where

$$
\delta=\frac{2 k\left[\eta \mu_{1}(1+a z)+\frac{1}{2}\left\{P_{1}+\left(P_{1} b-g \rho\right) z\right\}\right]}{\left[\eta \mu_{1} a+\frac{1}{2}\left(P_{1} b-g \rho\right)\right]}
$$

in equation (15), it may be reduced to

$$
\frac{d^{2} \varphi_{1}(\delta)}{d \delta^{2}}+\left(\frac{1}{4 \delta^{2}}+\frac{R}{\delta}-A\right) \varphi_{1}(\delta)=0
$$


where

$$
\begin{aligned}
R= & \frac{\frac{P_{1}}{2 \mu_{1}}+\frac{c^{2}}{c_{1}^{2}}}{2\left\{\eta\left(\frac{a}{k}\right)+\frac{1}{2}\left(\frac{P_{1}}{\mu_{1}} \frac{b}{k}-G\right)\right\}} \\
& -\frac{\frac{1}{2}\left(\frac{P_{1}}{2 \mu_{1}}\right)\left(\frac{b}{k}\right)\left(\eta+\frac{P_{1}}{2 \mu_{1}}\right)}{\left\{\eta\left(\frac{a}{k}\right)+\frac{1}{2}\left(\frac{P_{1}}{\mu_{1}} \frac{b}{k}-G\right)\right\}^{2}}, \\
A= & \frac{1}{4}-\frac{\frac{1}{2}\left(\frac{P_{1}}{2 \mu_{1}}\right)\left(\frac{b}{k}\right)}{\left\{\eta\left(\frac{a}{k}\right)+\frac{1}{2}\left(\frac{P_{1}}{\mu_{1}} \frac{b}{k}-G\right)\right\}}
\end{aligned}
$$

$c=(\omega / k)^{1 / 2}$ is the velocity of the torsional surface wave. $c_{1}=\left(\mu_{1} / \rho_{1}\right)^{1 / 2}$ is the velocity of the shear wave in the half-space. $G=\left(g \rho / \mu_{1} k\right)$ is Biot's gravity parameter.

Equation (16) is known as the Whittaker's equation, whose solution may be given by (Whittaker and Watson 1990)

$$
\begin{aligned}
\varphi_{1}(\delta)= & D_{1} W_{(R / 2 \sqrt{A}), 0}(2 \sqrt{A} \cdot \delta) \\
& +D_{2} W_{-(R / 2 \sqrt{A}), 0}(-2 \sqrt{A} \cdot \delta) .
\end{aligned}
$$

Since the solution should vanish at $z \rightarrow \infty$ (i.e., for $\delta \rightarrow \infty)$, we may take the solution as:

$$
\varphi_{1}(\delta)=D_{1} W_{(R / 2 \sqrt{A}), 0}(2 \sqrt{A} \cdot \delta) .
$$

Expanding the Whittaker function up to linear terms, equation (13) takes the form

$$
\begin{aligned}
v= & v_{1} \text { (say) } \\
= & \frac{D_{1} e^{-\sqrt{A} \delta}(2 \sqrt{A} \cdot \delta)^{\frac{R}{2 \sqrt{A}}}}{\left[\eta \mu_{1}(1+a z)+\frac{1}{2}\left\{P_{1}+\left(P_{1} b-g \rho\right) z\right\}\right]^{1 / 2}} \\
& \times\left\{1-\frac{\left(\frac{R}{2 \sqrt{A}}-\frac{1}{2}\right)^{2}}{2 \sqrt{A} \delta}\right\} J_{1}(k r) e^{i \omega t} .
\end{aligned}
$$

\section{Boundary conditions}

The following boundary conditions must be satisfied

(i) At the free surface $z=-H$, the stress is vanishing so that

$$
v_{0}=0 \quad \text { at } z=-H .
$$

(ii) The continuity of the displacement requires that

$$
v_{0}=v_{1} \quad \text { at } z=0 .
$$

(iii) At the interface $z=0$, the continuity of the stress requires that

$$
L_{0} \frac{\partial v_{0}}{\partial z}=\mu_{1} \frac{\partial v_{1}}{\partial z} \quad \text { at } z=0
$$

Using boundary condition (i), equation (9) reduces to equation (18)

$$
A_{1} e^{\left(m_{1}+\frac{1}{2 \alpha}\right) H}+A_{2} e^{-\left(m_{1}-\frac{1}{2 \alpha}\right) H}=0 .
$$

Using boundary condition (ii) in equation (9) and equation (17), we get equation (19)

$$
A_{1} \frac{1}{\sqrt{L_{0}}}+A_{2} \frac{1}{\sqrt{L_{0}}}=D_{1} \cdot S_{1}
$$

where

$$
S_{1}=\frac{e^{-\sqrt{A} \delta}(2 \sqrt{A} \delta)^{\frac{R}{2 \sqrt{A}}}}{\left(\eta \mu_{1}+\frac{P_{1}}{2}\right)^{1 / 2}}\left\{1-\frac{\left(\frac{R}{2 \sqrt{A}}-\frac{1}{2}\right)^{2}}{2 \sqrt{A} \delta}\right\} .
$$

Using boundary condition (iii) in equation (9) and equation (17), we get equation (20).

$$
\begin{aligned}
- & \sqrt{L_{0}}\left(m_{1}+\frac{1}{2 \alpha}\right) A_{1}+\sqrt{L_{0}}\left(m_{1}-\frac{1}{2 \alpha}\right) A_{2} \\
= & \mu_{1} D_{1} S_{2}
\end{aligned}
$$

where

$$
\begin{aligned}
& S_{2}=-e^{-\sqrt{A} \delta} \cdot 2 k \sqrt{A} \cdot\left(\eta \mu_{1}+\frac{P_{1}}{2}\right)^{-1 / 2} \\
& \times(2 \sqrt{A} \delta)^{\frac{R}{2 \sqrt{A}}}\left\{1-\frac{\left(\frac{R}{2 \sqrt{A}}-\frac{1}{2}\right)^{2}}{2 \sqrt{A} \delta}\right\} \\
& +2 k R e^{-\sqrt{A} \delta}\left(\eta \mu_{1}+\frac{P_{1}}{2}\right)^{-1 / 2}(2 \sqrt{A} \delta)^{\frac{R}{2 \sqrt{A}}-1} \\
& \times\left\{1-\frac{\left(\frac{R}{2 \sqrt{A}}-\frac{1}{2}\right)^{2}}{2 \sqrt{A} \delta}\right\} \\
& +e^{-\sqrt{A} \delta} \cdot 2 k \cdot\left(\eta \mu_{1}+\frac{P_{1}}{2}\right)^{-1 / 2}(2 \sqrt{A} \delta)^{\frac{R}{2 \sqrt{A}}} \\
& \times\left\{\frac{\left(\frac{R}{2 \sqrt{A}}-\frac{1}{2}\right)^{2}}{2 \sqrt{A} \delta^{2}}\right\} \\
& -\frac{1}{2} e^{-\sqrt{A} \delta}\left(\eta \mu_{1}+\frac{P_{1}}{2}\right)^{-3 / 2}(2 \sqrt{A} \delta)^{\frac{R}{2 \sqrt{A}}} \\
& \times\left\{\eta \mu_{1} a+\frac{1}{2}\left(P_{1} b-g \rho\right)\right\}\left\{1-\frac{\left(\frac{R}{2 \sqrt{A}}-\frac{1}{2}\right)^{2}}{2 \sqrt{A} \delta}\right\}
\end{aligned}
$$


Eliminating $A_{1}, A_{2}$ and $D_{1}$ using equations (18)(20), we get

$$
\left|\begin{array}{ccc}
e^{\left(m_{1}+\frac{1}{2 \alpha}\right) H} & e^{-\left(m_{1}-\frac{1}{2 \alpha}\right) H} & 0 \\
\frac{1}{\sqrt{L_{0}}} & \frac{1}{\sqrt{L_{0}}} & S_{1} \\
-\sqrt{L_{0}}\left(m_{1}+\frac{1}{2 \alpha}\right) & \sqrt{L_{0}}\left(m_{1}-\frac{1}{2 \alpha}\right) & \mu_{1} S_{2}
\end{array}\right|=0
$$

On expanding the determinant, we get

$$
\begin{gathered}
\cot \left(k H \sqrt{\frac{N_{0}}{L_{0}}\left(\frac{c^{2}}{c_{0}^{2}}-1\right)-\frac{1}{4 \alpha^{2} k^{2}}}\right) \\
=\frac{\frac{1}{2 \alpha k}+\frac{\mu_{1}}{L_{0}}\left(\frac{S_{2}}{S_{1} k}\right)}{\sqrt{\frac{N_{0}}{L_{0}}\left(\frac{c^{2}}{c_{0}^{2}}-1\right)-\frac{1}{4 \alpha^{2} k^{2}}}}
\end{gathered}
$$

where

$$
\begin{aligned}
\frac{S_{2}}{S_{1} k}= & -2 \sqrt{A}+\frac{R}{\sqrt{A} \delta}+\frac{2\left(\frac{R}{2 \sqrt{A}}-\frac{1}{2}\right)^{2}}{\delta\left\{2 \sqrt{A} \delta-\left(\frac{R}{2 \sqrt{A}}-\frac{1}{2}\right)^{2}\right\}} \\
& -\frac{1}{2} \frac{\left[\eta\left(\frac{a}{k}\right)+\left(\frac{1}{2} \frac{P_{1}}{\mu_{1}}\right)\left(\frac{b}{k}\right)-\frac{1}{2} G\right]}{\left(\eta+\frac{P_{1}}{2 \mu_{1}}\right)} .
\end{aligned}
$$

Equation (21) gives the required dispersion equation of torsional surface wave in an inhomogeneous anisotropic earth's crustal layer over a dry sandy mantle when the upper boundary assumed to be rigid.

\section{Particular cases}

Case I: When $(1 / \alpha) \rightarrow 0$, i.e., the directional rigidities and density becomes constant, then equation (21) reduces to

$$
\cot \left(k H \sqrt{\frac{N_{0}}{L_{0}}\left(\frac{c^{2}}{c_{0}^{2}}-1\right)}\right)=\frac{\mu_{1}\left(\frac{S_{2}}{S_{1} k}\right)}{L_{0} \sqrt{\frac{N_{0}}{L_{0}}\left(\frac{c^{2}}{c_{0}^{2}}-1\right)}}
$$

which is the dispersion equation of torsional surface wave in an anisotropic homogeneous layer under the effect of rigid boundary.

Case II: When $a \rightarrow 0$ and $b \rightarrow 0$, i.e., when the half-space is homogeneous under the effect of constant initial stress $P_{1}$, then the equation (21) becomes

$$
\begin{gathered}
\cot \left(k H \sqrt{\frac{N_{0}}{L_{0}}\left(\frac{c^{2}}{c_{0}^{2}}-1\right)-\frac{1}{4 \alpha^{2} k^{2}}}\right) \\
=\frac{\frac{1}{2 \alpha k}+\frac{\mu_{1}}{L_{0}} B_{1}}{\sqrt{\frac{N_{0}}{L_{0}}\left(\frac{c^{2}}{c_{0}^{2}}-1\right)-\frac{1}{4 \alpha^{2} k^{2}}}}
\end{gathered}
$$

where

$$
\begin{aligned}
B_{1} & =-1+\frac{1}{2} \frac{\left(\frac{P_{1}}{2 \mu_{1}}+\frac{c^{2}}{c_{1}^{2}}\right)}{\left(\eta+\frac{P_{1}}{2 \mu_{1}}\right)} \\
& +\frac{\left(\frac{P_{1}}{2 \mu_{1}}+\frac{c^{2}}{c_{1}^{2}}+\frac{G}{2}\right)^{2}}{2\left(\eta+\frac{P_{1}}{2 \mu_{1}}\right)\left\{4\left(\eta+\frac{P_{1}}{2 \mu_{1}}\right)+\frac{1}{G}\left(\frac{P_{1}}{2 \mu_{1}}+\frac{c^{2}}{c_{1}^{2}}+\frac{G}{2}\right)^{2}\right\}} \\
& +\frac{1}{4} \frac{G}{\left(\eta+\frac{P_{1}}{2 \mu_{1}}\right)}
\end{aligned}
$$

which is a dispersion equation of torsional surface wave in an inhomogeneous anisotropic layer over a homogeneous isotropic half-space when upper boundary plane is rigid.

Case III: When $\alpha \rightarrow \infty$ and $N_{0}=L_{0}$, i.e., the layer is homogeneous and isotropic

$$
\cot \left(k H \sqrt{\left(\frac{c^{2}}{c_{0}^{2}}-1\right)}\right)=\frac{\mu_{1}}{L_{0}} \frac{\left(\frac{S_{2}}{S_{1} k}\right)}{\sqrt{\left(\frac{c^{2}}{c_{0}^{2}}-1\right)}} .
$$

This gives the dispersion equation of torsional surface wave in a homogeneous isotropic layer over a dry sandy mantle.

Case IV: When $\alpha \rightarrow \infty, a \rightarrow 0, P_{1} \rightarrow 0$ and $N_{0}=$ $L_{0}$, i.e., the layer and the half-space both are homogeneous

$$
\cot \left(k H \sqrt{\left(\frac{c^{2}}{c_{0}^{2}}-1\right)}\right)=\frac{\mu_{1}}{L_{0}} \frac{\sqrt{\left(\frac{c^{2}}{c_{1}^{2}}-1\right)}}{\sqrt{\left(1-\frac{c^{2}}{c_{0}^{2}}\right)}},
$$

which is a well known classical equation Love wave under the effect of rigid boundary (Love 1944).

\section{Numerical results and discussion}

Based on the dispersion equation (21), numerical results are provided to show the propagation 
Table 1. Values of various dimensionless parameters.

\begin{tabular}{lccccccc}
\hline & Figure 2 & Figure 3 & Figure 4 & Figure 5 & Figure 6 & Figure 7 & Figure 8 \\
\hline$P_{1} / 2 \mu_{1}$ & - & - & 0.2 & 0.2 & 0.2 & 0.4 & 0.4 \\
$a / k$ & 0.2 & 0.2 & - & 0.2 & 0.2 & 0.2 & 0.2 \\
$b / k$ & 1.5 & 1.5 & 1.5 & - & 1.5 & 1.5 & 1.5 \\
$\alpha k$ & 1.2 & 1.2 & 1.2 & 1.2 & - & 1.2 & 1.2 \\
$G$ & 0.5 & 0.5 & 0.5 & 0.5 & 0.5 & - & 0.5 \\
$\eta$ & 1.0 & 1.0 & 1.0 & 1.0 & 1.0 & 1.0 & - \\
\hline
\end{tabular}

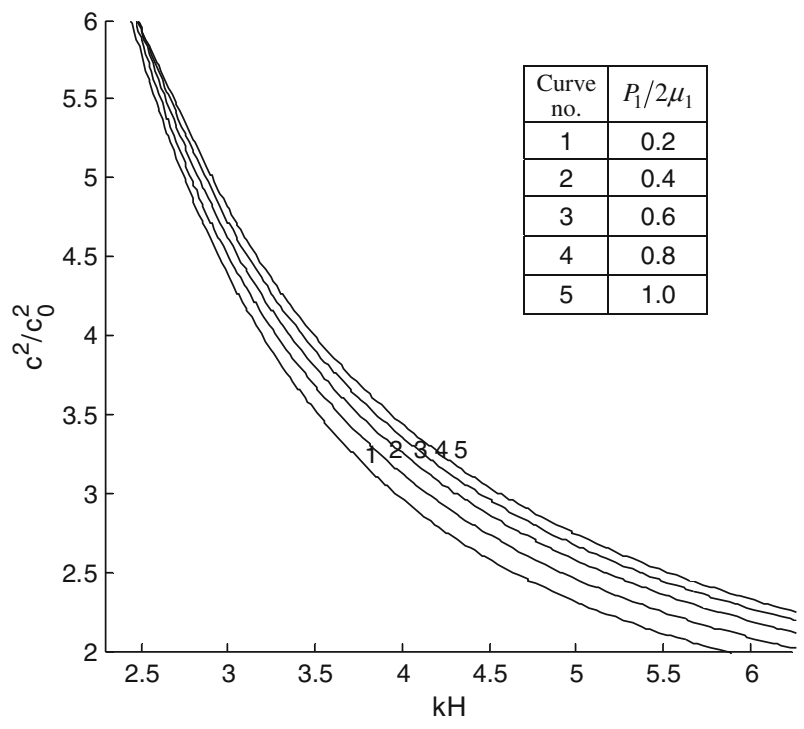

Figure 2. Dimensionless phase velocity vs. dimensionless wave number demonstrating the influence of compressive initial stress.

characteristics of torsional surface wave in the considered layer over a dry sandy mantle under the effect of gravity, initial stress and rigid boundary. The expansion of Whittaker function is taken as $W_{R, 0}(z)=e^{-z / 2} z^{R}\left\{1-(R-0.5)^{2} / 1 ! z\right\}$. The numerical data of various elastic constants and parameters have been taken from Gubbins (1990). In all the figures, curves have been plotted with vertical axis as dimensionless phase velocity $\left(c_{0}^{2} / c_{1}^{2}\right)$ against horizontal axis as dimensionless wave number $k H$. It has been found that with the increase of wave number, the phase velocity decreases rapidly in each of the figures under the considered values of various parameters. The main attention is paid on the influence of sandy parameter $(\eta)$ and Biot's gravity parameter $(G)$ along with the effect of inhomogeneity factor $\alpha k, a / k, b / k$ and dimensionless initial stress $\left(P_{1} / 2 \mu_{1}\right)$ (as given in table 1$)$. The values of $\left(c_{0}^{2} / c_{1}^{2}\right), N_{0} / L_{0}$ and $\mu_{1} / L_{0}$ have been taken as $0.2,0.7$ and 1.8 , respectively in all the figures.

Figure 2 discusses the dispersion curves for torsional surface wave when compressive initial stress $\left(P_{1} / 2 \mu_{1}>0\right)$ has been taken into account in the

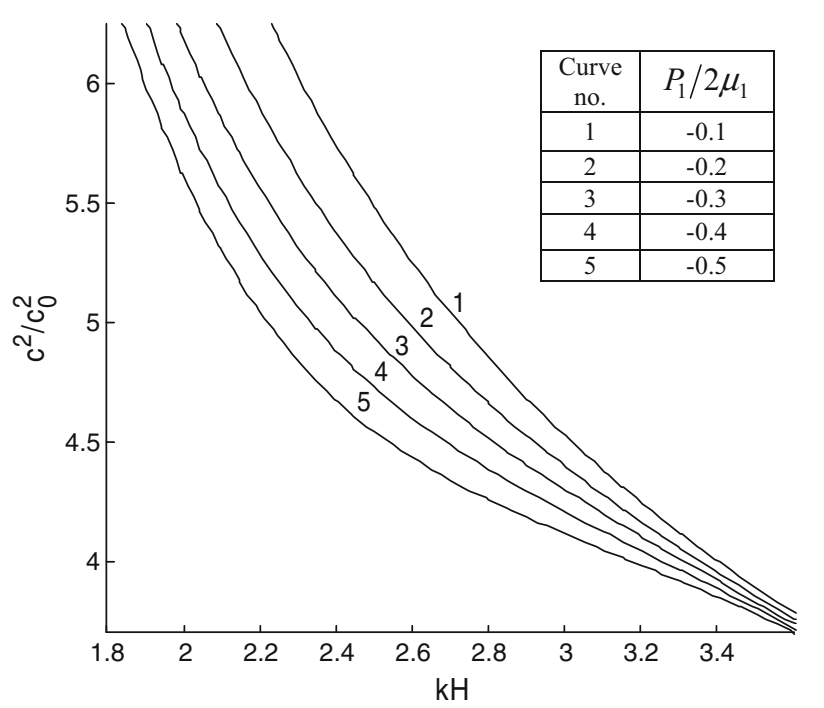

Figure 3. Dimensionless phase velocity vs. dimensionless wave number demonstrating the effect of tensile initial stress.

dry sandy mantle under the effect of gravity and rigid boundary. The value of $P_{1} / 2 \mu_{1}$ for curve no. 1 , no. 2 , no. 3 , no. 4 and no. 5 has been taken as $0.2,0.4,0.6,0.8$ and 1.0 , respectively. Following observations and effects are obtained under the above-considered values:

- As the compressive initial stress increases, the dimensionless phase velocity $\left(c_{0}^{2} / c_{1}^{2}\right)$ increases at a particular wave number.

- The curves are accumulating at $k H=2.5$ showing that although the compressive initial stress varies, velocity remains constant at that particular wave number.

- The curves being little far apart from each other at lower phase velocity reveal that compressive initial stress has much dominance at large values of wave number.

Figure 3 shows the effect of tensile initial stress $\left(P_{1} / 2 \mu_{1}<0\right)$ in the dry sandy mantle under the effect of gravity and rigid boundary. Here, the value of tensile initial stress $P_{1} / 2 \mu_{1}$ for curve no. 1 , no. 2 , no. 3 , no. 4 and no. 5 have been taken as -0.1 , 
$-0.2,-0.3,-0.4$ and -0.5 , respectively. Following observations have been made:

- It has been observed that decrease in tensile initial stress decreases the velocity of torsional surface wave at the same frequency.

- Here, the curves seem converging at $k H=3.5$ thereby making the phase velocity constant at $c^{2} / c_{0}^{2}=3.5$ at various magnitude of tensile initial stress. Whereas its dominant effect is visible at higher phase velocity and lower wave number.

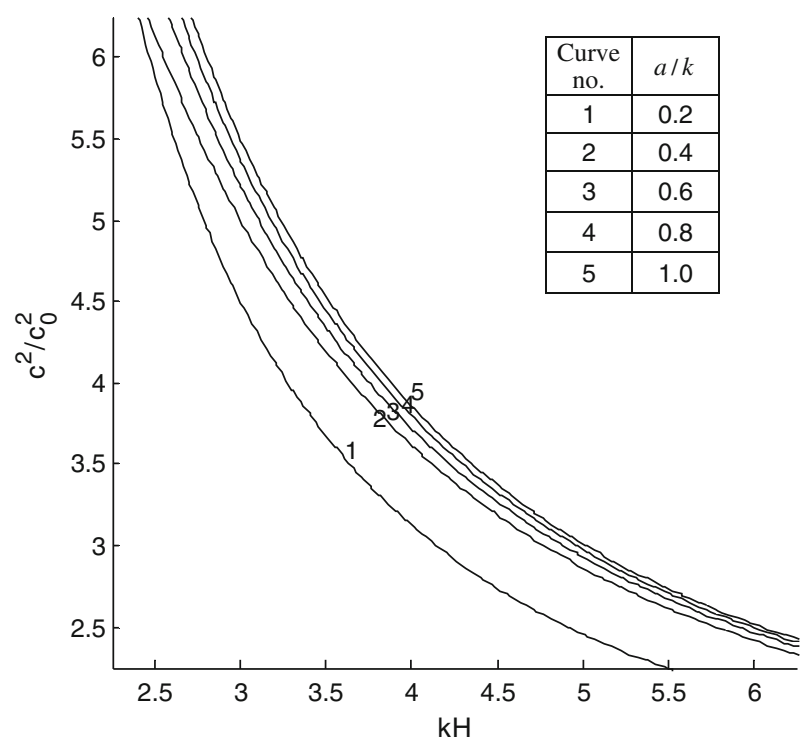

Figure 4. Dimensionless phase velocity vs. dimensionless wave number demonstrating the influence of inhomogeneity associated with rigidity of the half-space.

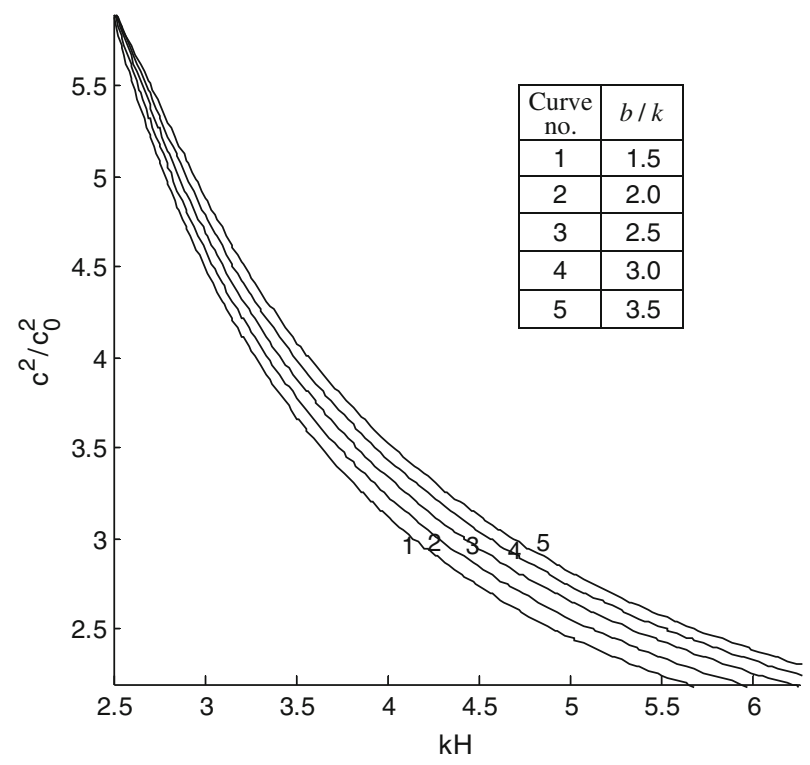

Figure 5. Dimensionless phase velocity vs. dimensionless wave number demonstrating the effect of inhomogeneity associated with initial stress of the half-space.
Figure 4 has been plotted to have a better understanding of the effect of the inhomogeneity parameter $(a / k)$ incorporated in the modulus of rigidity on the torsional wave velocity. The value of $(a / k)$ has been varied from 0.2 to 1 (i.e., 0.2 for curve no. 1, 0.4 for curve no. 2, 0.6 for curve no. $3,0.8$ for curve no. 4 and 1.0 for curve no. 5). We observed that

- As the dimensionless inhomogeneity factor $(a / k)$ increases, the phase velocity of torsional surface

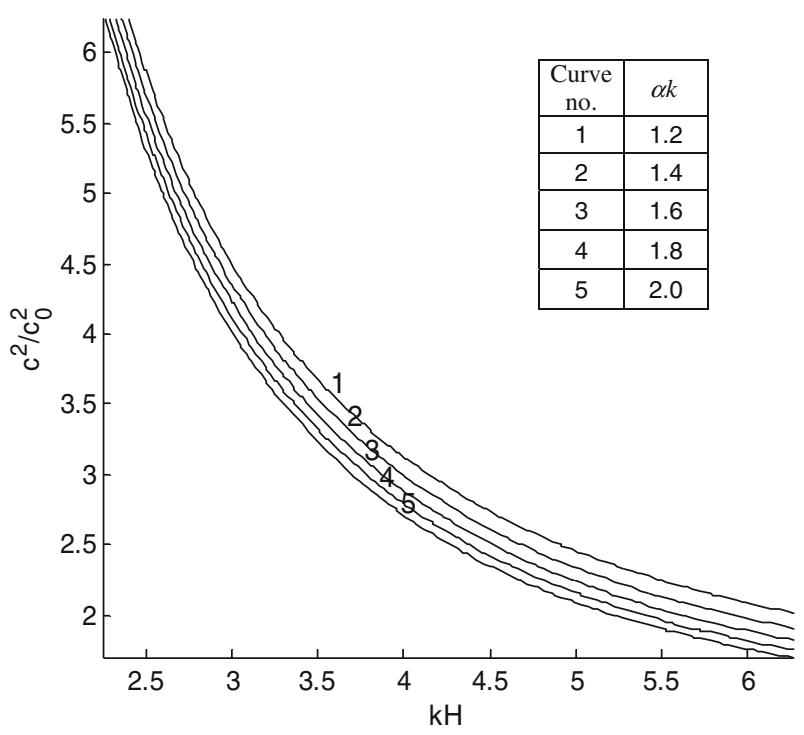

Figure 6. Dimensionless phase velocity vs. dimensionless wave number demonstrating the influence of inhomogeneity associated with the layer.

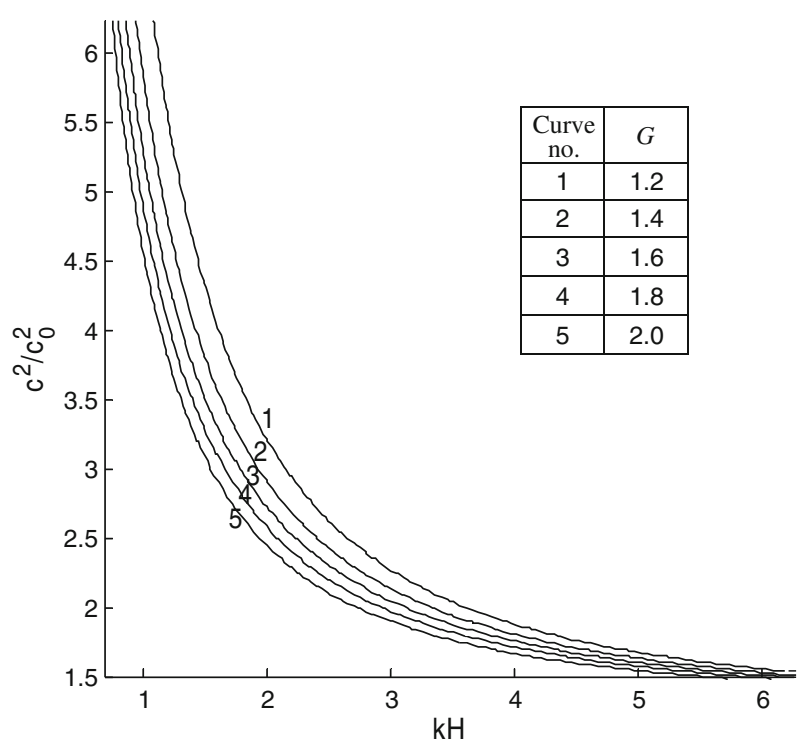

Figure 7. Dimensionless phase velocity vs. dimensionless wave number demonstrating the effect of Biot's gravity parameter. 
wave at a particular frequency also increases, which justifies the fact that velocity of the surface wave is directly proportional to the rigidity of the medium.

- The curves shift closer to each other as the value of $(a / k)$ increases, reveals that it has a negligible effect for the higher magnitude.

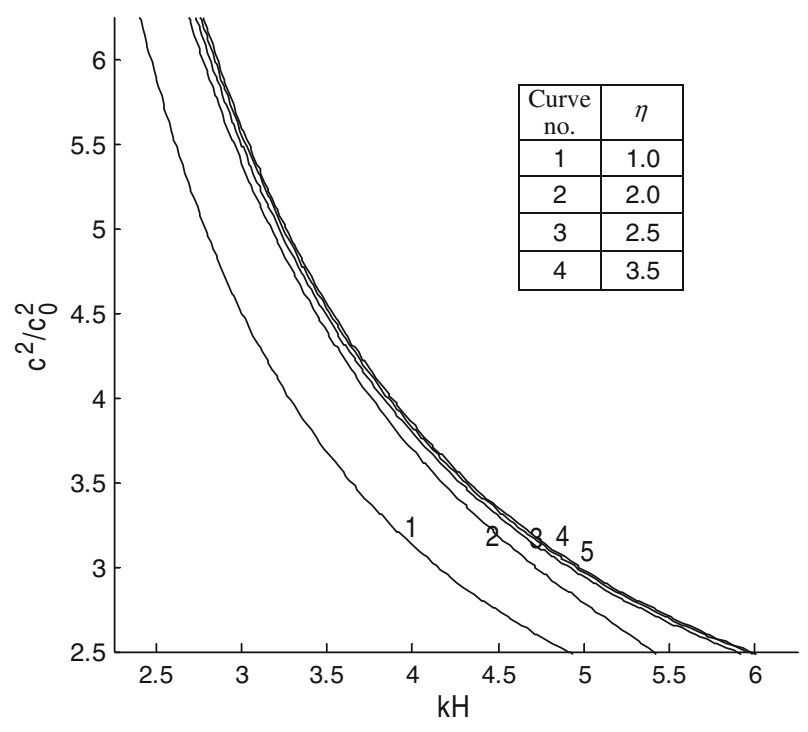

Figure 8. Dimensionless phase velocity vs. dimensionless wave number demonstrating the influence of sandy parameter.
In figure 5, an attempt has been made to come out with effect of inhomogeneity factor $(b / k)$. The value of $(b / k)$ for curve no. 1 , no. 2 , no. 3 , no. 4 and no. 5 are considered as 1.5, 2.0, 2.5, 3.0 and 3.5. It has been found that:

- Torsional wave velocity increases for the increasing value of $(b / k)$ at a particular frequency, prominent effect of which is visible at higher frequency.

- Similar to figure 2, here also the curves are accumulating at a single point denoting although inhomogeneity parameter $b / k$ varies, phase velocity remains constant for the same frequency.

Figure 6 signifies the effect of inhomogeneity factor $\alpha k$ present in the directional rigidities and density of the layer. The value of $\alpha k$ for curve no. 1, no. 2 , no. 3 , no. 4 and no. 5 has been taken as $1.2,1.4$, 1.6, 1.8 and 2.0, respectively. Following conclusions may be drawn from the figure:

- These curves show that the phase velocity of torsional surface wave decreases remarkably as the value of $\alpha k$ increases from 1.2 to 2.0.

- The curvature of the curves being equally apart shows the predominant influence of the inhomogeneity parameter associated with the layer.

Figure 7 shows the study of phase velocity under a wide range of Biot's gravity parameter $G$. Here

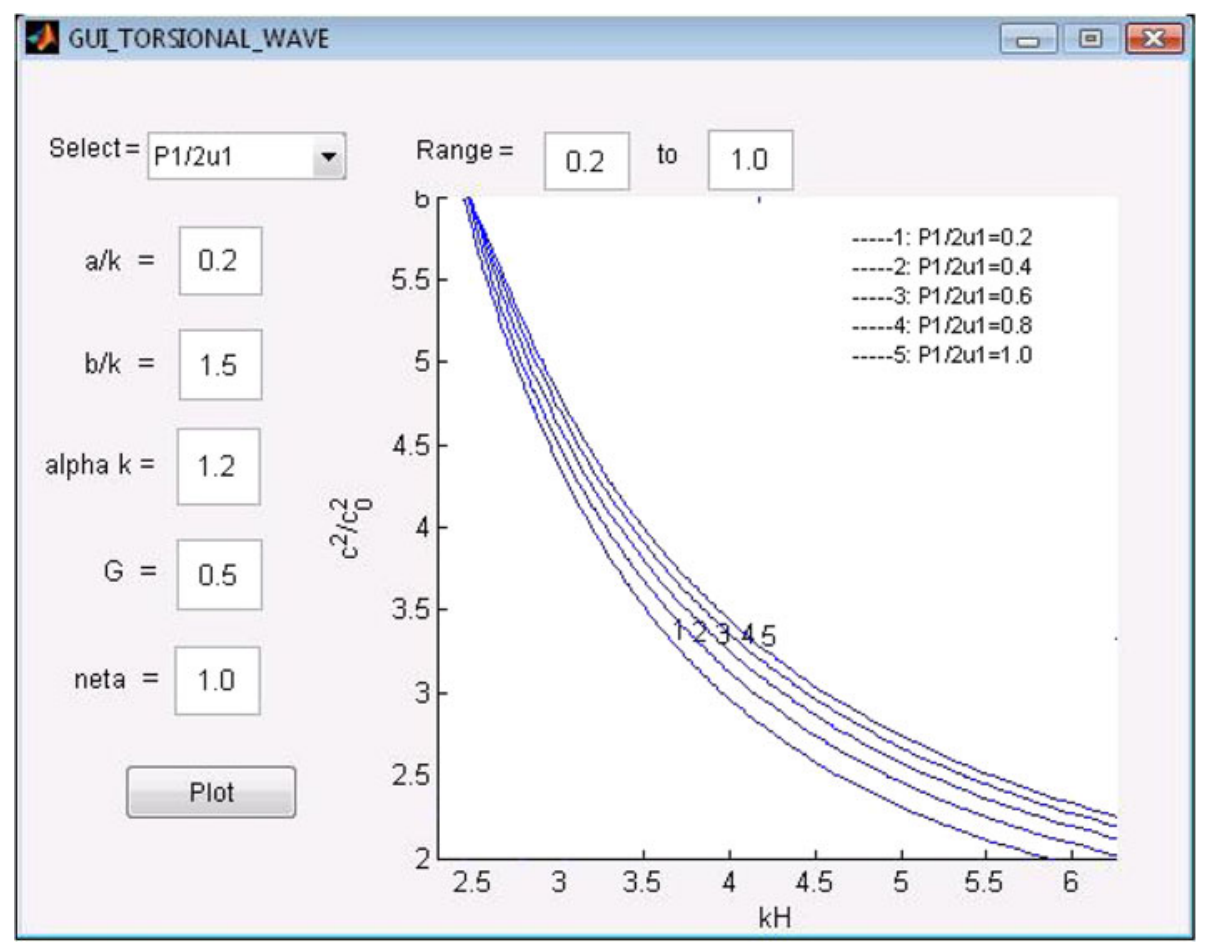

Figure 9. Snapshot of graphical user interface (GUI) developed in MATLAB 7.5. 
the value of $G$ for curve no.1, no. 2 , no. 3 , no. 4 and no. 5 are taken as 1.2, 1.4, 1.6, 1.8 and 2.0, respectively. It has been observed that

- The phase velocity of torsional surface wave in the layer increases significantly as the value of $G$ increases in the above-mentioned range.

- The effect of dimensionless gravity parameter $G$ almost vanishes as it approaches $k H=6.0$.

- The curves being steeper between $k H=1.0$ and 3.0 shows that $G$ has a perfect influence over the phase velocity of torsional surface wave.

Figure 8 describes the effect of sandy parameter $\eta$ on the velocity of torsional surface wave for the various values of $\eta$, i.e., 1.0, 2.0, 2.5 and 3.5 for curve nos. $1-4$, respectively. Following results may be drawn:

- The curves reflect that in the presence of compressive initial stress and the rigid boundary plane, as the value of sandy parameter $\eta$ increases, the phase velocity of torsional wave also increases.

- For $\eta=1$, the medium turn out to be perfect elastic thereby allowing torsional surface wave to propagate with least velocity as compared to other values of $\eta$ (i.e., 2.0, 2.5, 3.5) for which the medium becomes dry and sandy. Hence it can be concluded that under the above-considered value of various parameters, the possibility of torsional wave propagation in the layer is least when the half-space is elastic as compared to the case when the half-space is dry and sandy.

Figure 9 represents a screenshot of graphical user interface (GUI) software in MATLAB demonstrating the graph plotted in figure 2 as a sample. This GUI generalizes the finding of the present paper by allowing one to vary the ranges of different dimensionless parameter and also by providing different values to the various parameters involved. This will help one to observe the variation on the phase velocity of torsional surface wave against dimensionless wave number.

\section{Conclusion}

Propagation of torsional surface wave in an inhomogeneous anisotropic earth's crustal layer over a dry sandy mantle when upper boundary plane is rigid has been investigated analytically. The dispersion equation thus obtained in a closed form reduces into classical equation of Love wave when inhomogeneity parameters and initial stress is neglected thereby validating the solution of the problem discussed. We find that as the compressive initial stress increases, the phase velocity increases and as the tensile initial stress decreases the phase velocity also decreases. It is also found that the increasing value of inhomogeneity factor in rigidity and initial stress decreases the phase velocity of the torsional surface wave. The effect of Biot's gravity parameter has also come out prominently. It has been studied that as the value of Biot's gravity parameter increases, the velocity also increases. Study has also been made on sandy parameter and found that under a rigid boundary plane, torsional surface wave propagates with more ease when the half-space is dry and sandy rather than being elastic. Graphical user interface software has been developed in MATLAB to generalize the results of figures 2-8 so as to study the effect of compressive initial stress, tensile initial stress, inhomogeneity parameter and wave number on phase velocity of torsional wave with various sets of values. The present study may be useful for the study of seismic waves generated by artificial explosions.

\section{References}

Akbarov S D, Kepceler T and Egilmez M Mert 2011 Torsional wave dispersion in a finitely pre-strained hollow sandwich circular cylinder; J. Sound Vibr. 330 4519-4537.

Arora A and Tomar S K 2007 Elastic waves along a cylindrical borehole in a poroelastic medium saturated by two immiscible fluids; J. Earth Syst. Sci. 116(3) 225-234.

Biot M A 1965 Mechanics of Incremental Deformation; John Willey and Sons, New York.

Bullen K E 1940 The problem of the Earth's density variation; Bull. Seismol. Soc. Am. 30(3) 235-250.

Chattaraj R, Samal S K and Mahanti N C 2011 Propagation of torsional surface wave in anisotropic poroelastic medium under initial stress; Wave Motion 48 184-195.

Davini C, Paroni R and Puntle E 2008 An asymptotic approach to the torsional problem in thin rectangular domains; Meccanica 43(4) 429-435.

Dey S and Sarkar M G 2002 Torsional surface waves in an initially stressed anisotropic porous medium; J. Eng. Mech. 128(2) 184-189.

Dey S, Gupta A K and Gupta S 1996 Torsional surface waves in nonhomogeneous and anisotropic medium; Acoust. Soc. Am. 99(5) 2737-2741.

Dey S, Gupta A K and Gupta S 1998 Propagation of torsional surface waves in dry sandy medium under gravity; J. Math. Mech. Solid 3(2) 229-235.

Dey S, Gupta S, Gupta A K, Kar S K and De P K 2003 Propagation of torsional surface waves in an elastic layer with void pores over an elastic half-space with void pores; Tamkang J. Sci. Eng. 6(4) 241-249.

Georgiadis H G, Vardaulakis I and lykotrafitis G 2000 Torsional surface wave in gradient-elastic half-space; Wave Motion 31(4) 333-348.

Golamhossen F R 2000 Propagation of waves in an elastic cylinder with voids; Sci. Tech. Res. J. 5 43-52.

Gupta S, Majhi D K, Kundu S and Vishwakarma S K 2012a Propagation of torsional surface waves in a homogeneous layer of finite thickness over an initially stressed heterogeneous half-space; Appl. Math. Comput. 218 5655-5664. 
Gupta S, Majhi D K and Vishwakarma S K 2012b Torsional surface wave propagation in an initially stressed nonhomogeneous layer over a non-homogeneous half-space; Appl. Math. Comput. 219 3209-3218.

Gupta S, Vishwakarma S K, Majhi D K and Kundu S 2012c Influence of linearly varying density and rigidity on torsional surface waves in an inhomogeneous crustal layer; Appl. Math. Mech. 33(10) 1-14.

Gupta S, Majhi D K, Kundu S, Vishwakarma S K and Kundu S 2013 Possibility of Love wave propagation in a porous layer under the effect of linearly varying directional rigidities; Appl. Math. Model. 37 6652-6660.

Gubbins D 1990 Seismology and Plate Tectonics; Cambridge University Press, Cambridge/New York, 170p.

Kepceler T 2010 Torsional wave dispersion relation in a pre-stressed bi-material compounded cylinder with an imperfect interface; Appl. Math. Modelling 34 4058-4073.

Lesan D and Nappa L 2003 Axially symmetric problems for a porous elastic solid; Int. J. Solids Struct. 40(6) 5271-5286.

Love A E H 1944 A Treatise on Mathematical Theory of Elasticity; 4th edn, Dover Publication, New York.
Midya G K 2004 On Love-type surface waves in homogeneous micropolar elastic media; Int. J. Eng. Sci. 42(11-12) 1275-1288.

Ozturk A and Akbbarov S D 2009 Torsional wave propagation in a pre-stressed circular cylinder embedded in a pre-stressed elastic medium; Appl. Math. Model. 33 3636-3649.

Quintanilla R 2001 On uniqueness and continuous dependence in the nonlinear theory of mixtures of elastic solids with voids; Math. Mech. Solids 6(3) 281-298.

Sari C and Salk M 2002 Analysis of gravity anomalies with hyperbolic density contrast: An application to the gravity data of Western Anatolia; J. Balkan Geophys. Soc. 5(3) $87-96$.

Selim M M 2007 Propagation of torsional surface wave in heterogeneous half-space with irregular free surface; Appl. Math. Sci. 1(29-32) 1429-1437.

Vardoulakis I 1984 Torsional surface wave in inhomogeneous elastic media; Int. J. Numer. Anal. Methods Geomech. 8 $287-296$.

Whittaker E T and Watson G N 1990 A Course in Modern Analysis; 4th edn, Cambridge University Press, Cambridge. 